'SL Hardoon, ${ }^{2} \mathrm{PH}$ Whincup, 'I Petersen, 'RW Morris. 'Department of Primary Care \& Population Health, UCL, London, UK; '2Division of Population Health Sciences and Education, St George's, University of London, London, UK

Background Incidence of myocardial infarction (MI) in the UK general population has declined considerably in recent years. However it is unclear whether the decline in MI risk has occurred among people with diabetes. People with diabetes have an estimated twofold excess risk of MI, compared to those without diabetes. A differential trend in MI incidence among diabetic patients could correspond to a rise or fall in this excess risk, which has implications for prognosis and management of diabetes. We compared recent trends in MI incidence among those with and without diabetes in a representative UK population sample, and estimated the excess risk of MI among diabetic patients in different calendar periods.

Methods The population sample comprised 2,927,137 patients (49\% men) aged 30 years and over, with no prior MI, from 434 general practices belonging to The Health Improvement Network (THIN) UK-wide primary care database in 1995-2008. Incidence of MI in 1995-1998 was compared with that 10 years later in 20052008. Rate ratios comparing incidence over these intervals were estimated from multi-level Poisson regression (patients nested in practices), with an indicator for time interval as a covariate, adjusting for age and gender, with practice as a random effect. An interaction between time interval and an indicator for diabetes was used to assess whether the rate ratios comparing intervals differed among those with and without diabetes, and equivalently whether the excess relative risk among diabetic patients has changed over time. Results In 1995-1998 age-standardised incidence rates for MI (per 1000 person years) among those without and with diabetes were 3.22 (95\% CI 3.10-3.34) and 9.56 (8.42-10.7) respectively. In 20052008 , corresponding incidence rates were 1.47 (1.44-1.50) and 4.43 (4.23-4.65). Among people without diabetes, the rate ratio comparing incidence in 2005-2008 with 1995-1998 was 0.46 (0.44-0.48). Among people with diabetes, the corresponding rate ratio was 0.31 (0.28-0.35), indicating a greater decline in MI incidence over the period ( $p<0.001$ for interaction between interval and diabetes). Correspondingly, the rate ratio comparing incidence among those with diabetes to those without diabetes was attenuated from 2.70 (2.42$3.02)$ in 1995-1998 to 1.90 (1.80-2.00) in 2005-2008. Gender-specific analyses revealed the attenuation of the relative risks to be significant among both women and men.

Conclusion The excess risk of MI among diabetic patients appears to be falling over time. However, despite their improved prognosis, people with diabetes remain at a considerable excess risk of MI, emphasizing the need for continued concerted efforts to manage diabetes.

\section{OP59 DO DEMANDS AND WORRIES FROM CLOSE SOCIAL RELATIONS INCREASE THE RISK OF SUBSEOUENT INCIDENT IHD HOSPITALIZATION? A 7 YEAR LONGITUDINAL STUDY OF MIDDLE-AGED DANISH MEN AND WOMEN}

doi:10.1136/jech-2012-201753.059

R Lund, NH Rod, K Thielen, U Christensen. Social Medicine, Institute of Public Health, University of Copenhagen, Copenhagen, Denmark

Background The association between support from social relations and ischemic heart disease is well described, however the possible hazardous effects of negative aspects of social relations on cardiovascular health are less well known. The purpose of the present study was to analyze the possible influence of negative aspects of social relations (NASR) at baseline on the risk of development of ischemic heart disease (IHD) defined as incident hospitalized cases of acute myocardial infarction and chronic IHD during 7 year follow-up. NASR were defined as demands or worries from partner, children, family, and friends.
Methods Participants were included in a questionnaire-based study in 2000 and were a random sample $(\mathrm{N}=6767)$ of Danish men and women aged 40 or 50 years by October 1st 1999 from the Danish Longitudinal Study on Work, Unemployment and Health. Data for the present study are based on baseline questionnaire data in 2000 and register linked data from the period 2000-2007 on hospitalization for IHD (ICD10: I21-25). Cases of IHD (I21-25) four years prior to baseline were excluded from the analyses. In total 127 new cases of IHD were identified during follow-up.

Results Men who always or often experienced worries or demands from their partner had an increased risk of incident IHD compared to those who seldom/never experienced worries and demands $\mathrm{HR}(95 \% \mathrm{CI})=2.28(1.14-4.53)$ adjusted for age, socioeconomic status, cohabitation status, depressive symptoms, smoking and emotional support from all social relations. There was no association between demands/worries from partner and risk of development of IHD among women. Both men and women who experienced frequent worries and demands from their family (other than partner and children) were at increased risk of IHD HR=1.76(1.10-2.81) adjusted for above mentioned covariates and gender. Demands and worries from children and friends were not associated with significantly increased risk of IHD although estimates were in the same direction as for demands/worries from partner and family.

Conclusion For men, frequent demands and worries from a partner seem to be associated with increased risk of incident IHD hospitalization during 7 year follow-up. Demands/worries from family are risk factors for both women and men. Adjustment for the level of social support from all social relations did not change these conclusions. These findings confirm earlier findings of an association between NASR and self-reported angina pectoris. The weaker findings for women may partly be explained by the substantially smaller number of cases in this middle-aged cohort.

\section{OP60 RISK OF FUTURE DEPRESSION IN PEOPLE WHO ARE OBESE BUT METABOLICALLY HEALTHY: THE ENGLISH LONGITUDINAL STUDY OF AGEING}

doi:10.1136/jech-2012-201753.060

M Hamer, GD Batty, M Kivimaki. Epidemiology and Public Health, UCL, London, UK

Background There is some evidence to suggest that obesity is a risk factor for the development of depression, although this is not a universal finding. This discordance might be ascribed to the existence of a 'healthy obese phenotype' - that is, obesity in the absence of the associated burden of cardio-metabolic risk factors. We examined whether the association of obesity with depressive symptoms is dependent on the individual's metabolic health.

Methods Participants were 3851 men and women (aged $63.0 \pm 8.9$ yrs, $45.1 \%$ men) from the English Longitudinal Study of Ageing, a prospective study of community dwelling older adults. Obesity was defined as body mass index $\geq 30 \mathrm{~kg} / \mathrm{m}^{2}$. Based on blood pressure, HDL-cholesterol, triglycerides, glycated haemoglobin, and C-reactive protein, participants were classified as 'metabolically healthy' ( 0 or 1 metabolic abnormality) or 'unhealthy' ( $\geq 2$ metabolic abnormalities). Depressive symptoms were assessed at baseline and at 2 years follow up using the 8-item Centre of Epidemiological Studies Depression (CES-D) scale.

Results Obesity prevalence was $27.5 \%$, but $34.3 \%$ of this group was categorized as metabolically healthy at baseline. Relative to non-obese healthy participants, after adjustment for baseline CES-D score and other covariates, the metabolically unhealthy obese participants had elevated risk of depressive symptoms at follow-up (odds ratio $[\mathrm{OR}]=1.50,95 \% \mathrm{CI}, 1.05-2.15$ ), although the metabolically healthy obese did not $(\mathrm{OR}=1.38,95 \% \mathrm{CI}, 0.88-2.17)$. We repeated the main analysis after excluding 451 participants with existing depressive symptoms $(C E S-D \geq 4)$ at baseline. There were 238 incident cases of depression at follow up, and in comparison 